\title{
The composition and dynamics of cell-substratum adhesions in locomoting
}

\section{fish keratocytes}

\author{
Juliet Lee ${ }^{1, *}$ and Ken Jacobson ${ }^{1,2}$ \\ ${ }^{1}$ Department of Cell Biology and Anatomy, and ${ }^{2}$ Lineberger Comprehensive Cancer Center, University of North Carolina, Chapel \\ Hill, NC, USA \\ *Author for correspondence
}

\section{SUMMARY}

Close contacts are the predominant type of cell-substratum adhesion in rapidly moving cells yet little is known about their composition and dynamics. To address these issues we have attempted to identify the molecular components of close contacts formed in rapidly moving fish epithelial keratocytes. In addition we have utilized the simple shape of keratocytes to explore the relationship between close contact formation and rapid locomotion.

$\beta 1$ Integrin and talin molecules were found to be localized within a narrow rim of very close contact along the leading edge. These molecules together with vinculin were also found within small foci distributed evenly throughout the lamella, corresponding to regions of variable close contact. $\alpha$-Actinin was found in foci within older, more posteriorly located regions of the lamella and along stress fibers. In addition to close contacts, small focal adhesion-like structures which stained positively for all antibodies tested were found at the tips of stress fibers within retracting cell margins.

Interference reflection and total internal reflection microscopy of moving keratocytes showed cell-substratum contacts to be organized into distinct patterns that appear to move forwards, in concert with the leading edge. A feature common to all cells is a rim of very close contact at the leading edge. This region is specialized for the

\section{INTRODUCTION}

The formation of adhesive contacts between the ventral cell surface and the substratum is essential for locomotion of cells along a surface. Cells generally make two kinds of adhesive contact with the substratum (Izzard and Lochner, 1976, 1980) as defined by interference reflection microscopy (IRM). Focal adhesions represent the closest approach of the ventral cell surface to the substratum (10-15 nm). These appear by IRM as black elongated structures, oriented in the direction of stress fibers. Close contacts are broad irregularly shaped regions that appear as varying shades of gray by IRM, corresponding to an $\sim 30-50 \mathrm{~nm}$ cell-substratum separation distance. The dynamics of these two types of structure also differ. Focal contacts remain stationary with respect to the substratum during cell movement whereas close contacts formation of new cell-substratum adhesions and is the site where patterns of close contact are generated. We have found that cell locomotion is most rapid when a uniform contact pattern is present but cell speed is progressively reduced as the contact pattern becomes more irregular. Furthermore, the local rate of lamellar extension is most rapid when underlain by regions of intermediate closeness to the substratum, but is reduced or ceases if the underlying contact is either very close or more distant, respectively.

Our results suggest that close contacts and focal adhesions are related structures formed from a common hierarchy of molecular interactions. In addition the relationship between close contact formation and lamellar extension indicates a direct coupling between these two processes at the leading edge. Furthermore, we can explain the dynamic behaviour of close contacts in terms of the relative rates of trapping and release of component molecules that is initiated at the leading edge. We suggest that regulation of the molecular dynamics involved in leading edge specialization determines both the pattern of cell-substratum contacts and the net rate of actin filament assembly.

Key words: Cell-substratum, Adhesion, Keratocyte, Immunofluorescence, IRM (interference reflection microscopy) appear to advance in concert with the extending cell edge (Izzard and Lochner, 1980).

Of the two types of contact, focal adhesions have been the most extensively studied (Burridge et al., 1988). One reason for this is that the stability of these structures has facilitated their isolation and biochemical characterization. Another reason is that the association of focal adhesions with actin filament bundles suggested these regions were important for cell motility (Abercrombie et al., 1971; Heath and Dunn, 1978; Wehland et al., 1979). Recently, further impetus to the study of focal adhesions has come from the finding that they are sites of signal transduction (Hynes, 1992; Clark and Brugge, 1995; Schwartz et al., 1995). Although there have been some investigations into the composition of focal adhesion precursors (DePasquale and Izzard, 1987; Rinnerthaler et al., 1988) very little is known about the composition of close contacts. 
Despite the paucity of structural information about close contacts, there are many indications that close contacts are of greater importance for rapid cell locomotion than focal adhesions. Rapidly locomoting cell types such as amoebae, nerve growth cones, metastatic cells (Lackie, 1986) white blood cells (Armstrong and Lackie, 1975) epidermal cells and amphibian leukocytes (Radice, 1980; Kolega et al., 1982) form mainly close adhesive contacts with the substratum. Slower moving cell types, such as chick heart fibroblasts, may initially form few or no focal adhesions when freshly plated (Couchman and Rees, 1979). However, as focal adhesions begin to form, the rate of cell movement decreases. In fibroblasts, focal adhesion formation can occur independently from lamellar extension. Instead close contact formation has been shown to be necessary for lamellar extension, since this ceases when close contacts are lost but resumes when they are reformed (Izzard and Lochner, 1980).

Epithelial keratocytes of fish and amphibians are one of the most rapidly moving eukaryotic cell types. These cells form predominately close contacts with the substratum (Radice, 1980; Bereiter-Hahn et al., 1981; Kolega et al., 1982; Lee et al., 1993a). In addition lamellar extension and retraction in keratocytes is highly coordinated such that their simple, semicircular shape is maintained (Lee et al., 1993b). Since the kinematic principles underlying the movement of these cells has already been described, this allows us to better discern the relationship between adhesion formation and rapid locomotion. The study of simple-shaped cells may provide more insight into such a relationship than complex-shaped cells, whose rates of extension and retraction are not as highly coordinated.

The composition of close substratum contacts was investigated by immunofluorescence staining of fixed keratocytes using antibodies against known components of focal adhesions, such as $\beta 1$-integrin, talin, vinculin, $\alpha$-actinin, paxillin and the focal adhesion kinase (FAK). Close contacts were found to contain two or more of these molecules except for paxillin and FAK. Of particular interest is an outer rim of very close contact at the leading edge which is enriched in integrin and talin molecules. The dynamics of close contacts in moving keratocytes was observed using interference reflection microscopy (IRM) and total internal reflection microscopy (TIRF). It was found that the formation of a new close cellsubstratum contact occurs simultaneously with lamellar extension, within a narrow rim along the leading edge. During keratocyte locomotion, distinct patterns of cell-substratum contact, which consist of regions varying in closeness of approach to the substratum, emerge from the leading edge. Increasingly irregular patterns of close contact are associated with decreased rates of lamellar extension and overall cell speed together with a more irregular cell outline.

These results are discussed in relation to the trapping and release of adhesion components at the leading edge. This mechanism is consistent both with the formation of different types of cell-substratum contact and the generation of patterns at the leading edge.

\section{MATERIALS AND METHODS}

\section{Cell culture}

Fish epithelial keratocytes were cultured from the scales of gold fish
Carrasius auratus or Molly fish Mollensia sphenops, as described previously (Lee et al., 1993b). Briefly, scales were removed from the fish and washed three times, for 5 minutes, in cell culture medium RPMI 1640 supplemented with $10 \%$ fetal calf serum, penicillin/streptomycin $(0.1 \%)$ and Fungizone $(0.1 \%)$. Scales were sandwiched between two $22 \mathrm{~mm}^{2}$ square glass coverslips in $\sim 40 \mu \mathrm{l}$ of culture medium and left overnight in a humidified chamber at room temperature. Sheets of keratocytes that had migrated away from the scale could be disaggregated while remaining attached to the coverslip, by washing for 5 minutes in calcium free, phosphate buffered saline (PBS). In this way adequate numbers of single moving cells could be obtained. Cells were then replaced into culture medium until ready for use. Fish fibroblasts were obtained from fish scales, after 4 days to a week in culture as described above for keratocytes.

\section{Preparation of cells for video microscopy}

For IRM observations, keratocytes attached to a glass coverslip were washed three times in PBS and then mounted on a microscope slide by inverting the coverslip onto a drop of culture medium, centered between two strips of Parafilm, then sealing it with Valap. For in situ experiments, coverslips with attached keratocytes were mounted faceup in the well (16 mm diameter, $2.5 \mathrm{~mm}$ depth) of a metal slidechamber. In this way, the same group of keratocytes could be observed immediately before and after fixation. For TIRF, keratocytes were washed three times with PBS, for 1 minute each, and stained with 50 $\mu \mathrm{g} / \mathrm{ml}$ DiI (Molecular Probes, Eugene, Oregon, USA) in PBS for 30 minutes at room temperature. Cells were washed in PBS as above and mounted on a microscope slide in PBS.

\section{Immunofluorescence}

Primary antibodies to talin, vinculin, paxillin and $\alpha$-actinin were gifts from Dr K. Burridge. Anti-FAK antibodies were a gift from Dr L. Romer. Polyclonal anti- $\beta 1$-integrin antibodies were obtained from Chemicon International Inc. (Temecula, California, USA). All antibodies were polyclonal, except for anti-paxillin.

\section{Optimization of fixation procedure for fish keratocytes}

Since the cytoskeleton and cell-substratum adhesions of keratocytes are highly labile structures they are not well preserved by the usual methods of fixation such as $3.7 \%$ formaldehyde. To optimize the fixation protocol for keratocytes, IRM and immunofluorescence images were compared following different methods of fixation. In addition, to assess the effectiveness of various methods of fixation in preserving the IRM image of living cells, keratocytes were observed immediately prior to fixation in situ on the microscope stage. The degree of cell permeabilization that accompanies fixation or post processing for immunofluorescence was found to have marked, opposing effects on the quality of the fixed IRM and immunofluorescence images, respectively. As the degree of permeabilization is increased, the IRM image deteriorates because the amount of light reflected from the medium-cell interface is reduced, thus decreasing the contrast of the IRM image. However, the corresponding immunofluorescence image improves because increased permeabilization will facilitate access of antibodies to intracellular components and so enhance visualization of molecules within adhesion sites. Fixation protocols that lead to increasing degrees of permeabilization are as follows: (A) a mixture containing $2.5 \%$ glutaraldehyde, $0.025 \%$ Triton $\mathrm{X}-100$ and rhodamine phalloidin (1:300) in PBS for 40 seconds at $4{ }^{\circ} \mathrm{C}$; (B) absolute methanol at $-20^{\circ} \mathrm{C}$, for $40-60$ seconds; (C) $2.5 \%$ glutaraldehyde for 5 minutes at $4{ }^{\circ} \mathrm{C}$ followed by permeabilization in $0.5 \%$ Triton-X 100 for 1 minute at room temperature; (D) a mixture containing $1.25 \%$ glutaraldehyde and $0.5 \%$ Triton X-100 in PBS for 5 minutes at $4^{\circ} \mathrm{C}$. Of the above fixation protocols, A provides optimal preservation of keratocytes for paired IRM and immunofluorescence (Fig. 1). Fixation with methanol is also adequate, however, the pattern of close contacts is not preserved quite as well although immunolocalization of antibodies to contact regions tends to be more obvious than with protocol A.

Following fixation cells were gently washed in PBS three times, 

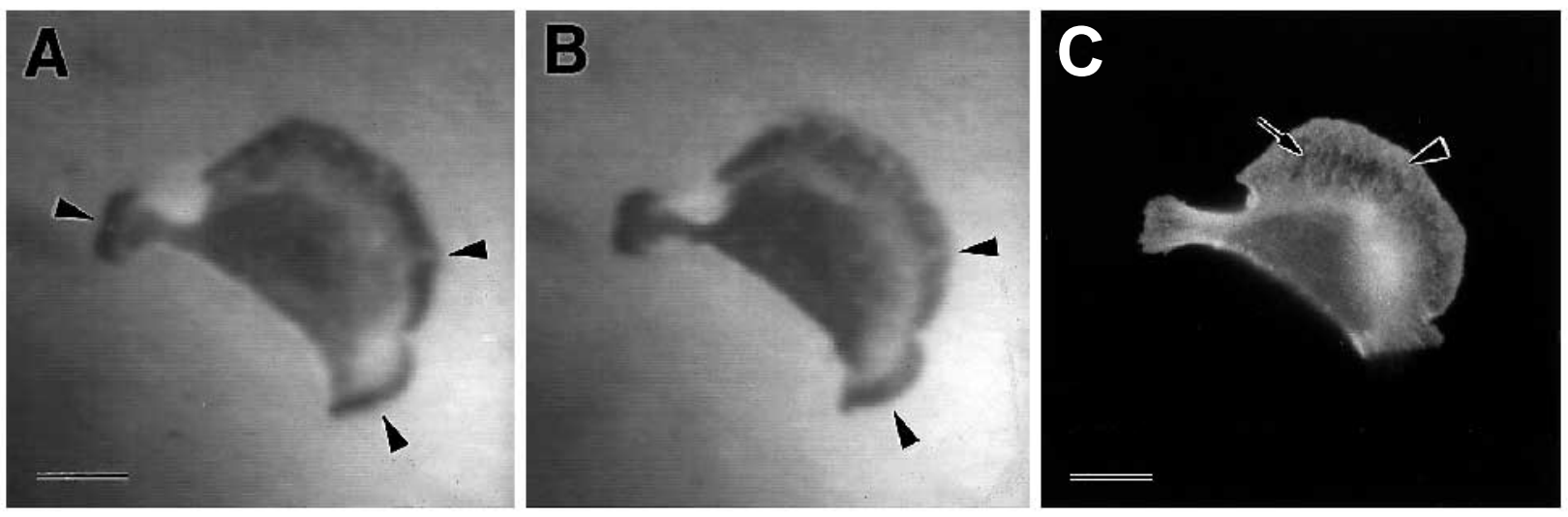

Fig. 1. Digitized video IRM images of a keratocyte prior to fixation (A) and immediately after 40 seconds of fixation (B) using a mixture of $2.5 \%$ glutaraldehyde, $0.025 \%$ Triton X-100 and rhodamine phalloidin (1:300). The pattern of contacts is virtually unchanged by this fixation protocol (arrowheads). Rhodamine phalloidin staining (C) of this cell, shows the actin cytoskeleton to be well preserved. This is indicated by the fibrillar appearance of the actin meshwork throughout the lamella (arrow), which is typically more dense toward the cell edge (arrowhead). Note that the lower edge of this cell is not visible due to accidental photobleaching of this region during a previous photographic exposure. Bar, $10 \mu \mathrm{m}$.

each for 1 minute. Cells were then washed with $1 \%$ bovine serum albumin for 15 minutes, prior to immunolabeling with primary and secondary antibodies. Cells were then mounted in an aqueous based anti-fading agent (Slowfade, Molecular Probes, Eugene, Oregon, USA) to allow visualization of the IRM image while protecting against excessive fluorescence photobleaching. Fish fibroblasts, which form typical focal adhesions as seen by IRM, were used in these studies as positive controls and to test cross-species reactivity of the antibodies. All antibodies tested colocalized within focal adhesions of fish fibroblasts (data not shown).

\section{Optical techniques}

Interference reflection microscopy (IRM) was performed on an inverted Nikon Diaphot fluorescence microscope according to the method of Bereiter-Hahn et al. (1979), using a Leitz $\times 100$ objective with a quartz $(\lambda / 4)$ wave plate, NA 1.32 . Immunofluorescence observations of labeled keratocytes were made using the same objective, focused at the plane of the substratum. Total internal reflection microscopy (TIRF) was performed according to the method of Reichert and Truskey (1990).

\section{Image acquisition and processing}

Video images were acquired using a low light level ISIT 66 camera (Dage-MTI, Inc., Michigan, Indiana, USA) in manual mode, together with Image 1 imaging software (Universal Imaging Corp., West Chester, Pennsylvania, USA). Images of moving cells or IRM/immunofluorescence pairs were stored on the computer (in digitized form) on video tape, or on optical discs using an optical disc recorder, TQ-2028F (Panasonic, Osaka, Japan). Cell speed was calculated from the average displacement size, taken at 1-2 second intervals for a period of about 2 minutes. Displacement data were obtained from the cell centroid coordinates of thresholded IRM images. Paired IRM and immunofluorescence images were recorded photographically, using a $35 \mathrm{~mm}$ camera (Nikon-FX35DX) equipped with an auto exposure meter and black and white film TMAX 400 (Kodak, Rochester, New York, USA).

\section{RESULTS}

\section{Types of cell-substratum adhesion in fish keratocytes}

The ventral cell surface makes varying degrees of close contact with the substratum (Fig. 2A-C) which appear by IRM as different shades of gray (Izzard and Lochner, 1976). Dark gray or black regions represent a cell-separation distance of 15-30 $\mathrm{nm}$ and are referred to by us as very close contacts. Gray to light gray regions (but darker than background) represent areas of intermediate approach to the substratum $(30-50 \mathrm{~nm})$ and are referred to as close contacts. Regions that appear white, termed distant contacts, represent the largest cell-substratum separation distance $(\sim 100 \mathrm{~nm})$. Areas where the cell is not in contact with the substratum $(>100 \mathrm{~nm})$ match the background gray. Close contacts are generally diffuse, irregularly shaped structures that vary in size and distribution beneath the lamella. However, regions of closest approach to the substratum are more well defined. In addition to close cell-substratum contacts, keratocytes can form focal adhesions. These appear by IRM as small dark streaks and are usually found perpendicular to the rearmost edges of the lamella where retraction is taking place (Fig. 2C).

\section{Keratocytes display distinct patterns of close contact}

The ventral surface of moving keratocytes consists mostly of a uniform region of intermediate close contact. However, individual contacts of variable closeness to the substratum can form patterns along the leading edge of the cell that can be described as falling into three broad categories. The simplest pattern consists of a dark gray rim of very close contact along the extending cell margin and a region of gray, intermediate contact beneath the remainder of the ventral surface (Fig. 2A). A more complex pattern consists of, in addition to an outer rim of very close contact, a periodic arrangement of very close and distant contact along the cell margin (Fig. 2A). The most complex pattern consists of adhesions of varying closeness that are irregular in size, shape and distribution along the extending cell margin (Fig. 2C). Cells can display one or more of these patterns in any sequence over a period of several minutes. Each pattern may persist for up to $\sim 2$ minutes.

\section{Observation of close cell-substratum adhesions by TIRF}

It has been shown previously that dark gray or black regions 

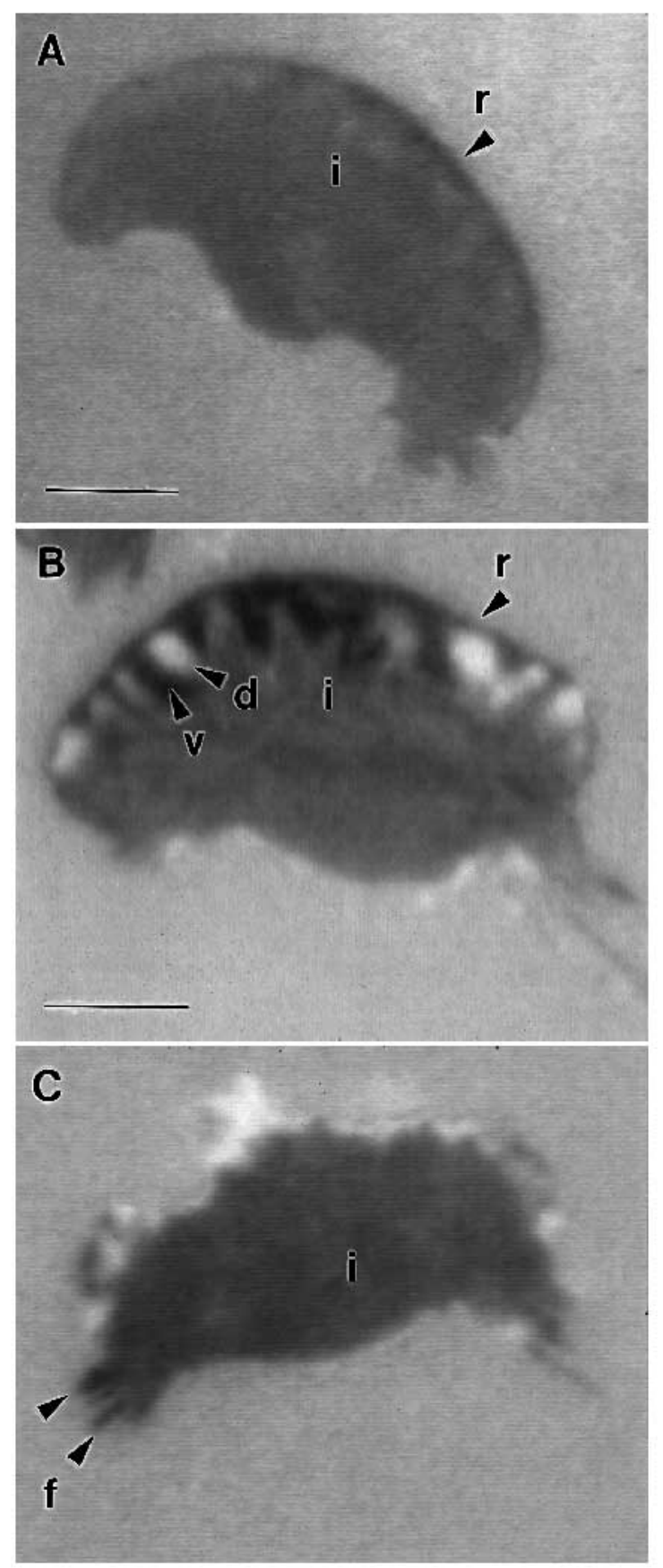

Fig. 2. IRM video images of moving keratocytes displaying three major categories of contact pattern. All cells possess a uniform region of intermediate close contact (i) extending over most of the ventral cell surface. Highly dynamic patterns of close contact are arranged along the leading cell edge. (A) The simple pattern consists of an outer rim (r) of very close contact and an intermediate region of close contact beneath the remainder of the ventral cell surface. (B) The periodic pattern consists of an outer rim (r) of very close contact skirting a crescent-shaped band of alternating very close (v) and distant contacts (d). The width of this band perpendicular to the cell margin, is greatest adjacent to regions of rapid lamellar extension (cell apex) and narrower where lamellar extension is slowest (rear cell edges). (C) Irregular patterns of close contact consist of a band of close contacts, along the leading cell edge whose size shape, and closeness of approach to the substratum varies. Focal adhesions (f) are often found at retracting cell margins, irrespective of contact pattern. Bar, $10 \mu \mathrm{m}$. viewed by IRM can be caused by the destructive interference of light reflected from both dorsal and ventral cell surfaces (Todd et al., 1988). Since the keratocyte lamella is only a few tenths of a micron in thickness it was important to show that the appearance of close contacts as varying shades of gray is due to their closeness of approach to the substratum and not to variations in thickness of the lamella. Moving keratocytes that had been stained with the lipid analog DiI were viewed with TIRF (Fig. 3) to see if patterns of close contact correspond to those seen by IRM. The advantage of this method is that it is not affected by the thickness of the lamella since the excitation of fluorescence is related only to closeness of the ventral surface to the substratum. Thus regions of closest contact will appear brighter than regions further away producing an image that is essentially the inverse of an IRM image. A typical periodic pattern of close contacts viewed by TIRF appears as an alternating pattern of bright and darker regions along the extending cell margin. A bright, outer rim of close contact can also be seen. These observations confirm that the IRM used for this study is a valid representation of cell-substratum separation distances.

\section{The composition of cell-substratum contacts}

Immunofluorescence staining of fish keratocytes (Figs 4 and 5) was performed using the following antibodies, against $\beta 1$ integrin, talin, vinculin, $\alpha$-actinin, paxillin and the focal adhesion kinase (FAK). Comparisons were made between IRM and immunofluorescence images in an attempt to identify the molecular components within different types of cell-substratum adhesion. $\beta 1$ integrin and talin molecules were found within small punctata dispersed throughout the lamella (Fig. 4D and E). In addition, a rim of increased fluorescence intensity exists along the leading edge of both anti- $\beta 1$ integrin and talin stained cells which corresponds to a dark gray region of very close cell-substratum contact, in the IRM images (compare Fig. 4A with D, and B with E). This is confirmed by the graphs of averaged line intensity scans for $\beta 1$ integrin and talin (Fig. 4G and H, respectively) taken along the rectangular regions in Fig. 4A and B. Vinculin (Fig. 4F) was found in small foci, distributed evenly throughout the lamella and in focal adhesions at retracting cell margins (Fig. 4C and F). However, unlike $\beta 1$ integrin and talin, vinculin is not enriched in the outer rim of very close contact (Fig. 4C,F and I). Staining for $\alpha$ actinin (Fig. 5A and B) is unusual in that it is excluded from a region approximately $5 \mu \mathrm{m}$ wide, behind the leading edge. Instead it is found in aggregates within more posterior regions of the lamella and along stress fibers. Although there are some differences in spatial distribution of the above molecules within the lamella, localization to specific regions of close contact could not be seen. Anti-FAK immunofluorescence was found predominately within regions corresponding to small elongated focal adhesions (Fig. 5C and D). FAK is also seen in small foci sparsely distributed throughout the lamella but not within the outer rim of very close contact. Paxillin was found exclusively at the termini of stress fibers within focal adhesions (Fig. 5E and F).

\section{Patterns of close contact are generated at the leading cell edge}

Time-lapse video recordings of moving keratocytes viewed by IRM show individual regions of close contact to be highly 

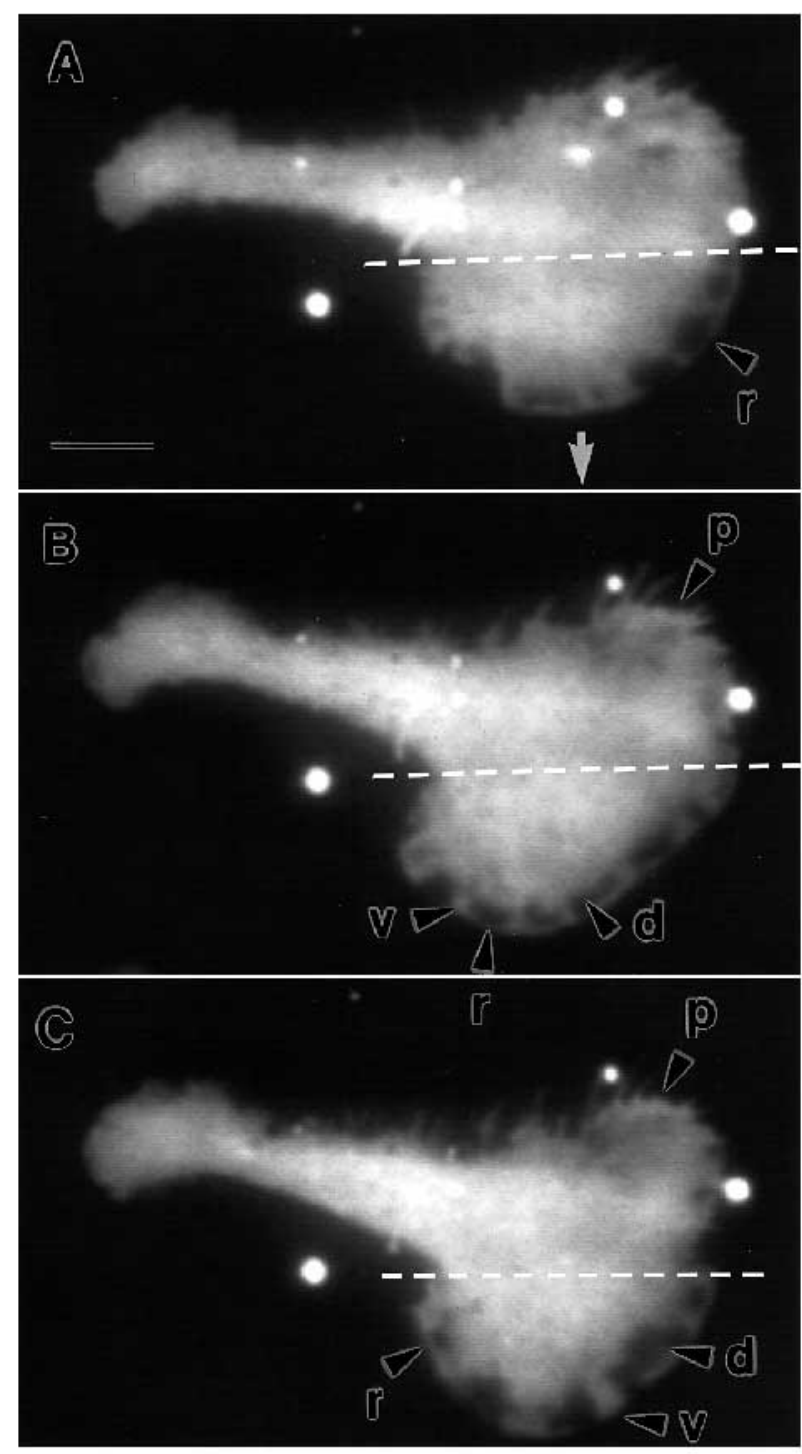

Fig. 3. (A-C) A sequence of TIRF images, acquired at approximately 40 second intervals, of a keratocyte stained with DiI. The cell remains tethered at its rear end while the lamella moves in the direction indicated (white arrow in A). The separation distance between the ventral cell surface and the substratum is inversely proportional to fluorescence intensity. A periodic pattern of close contact can be seen along the extending cell margin (below broken line). Bright regions (v) represent areas of close approach to the substratum. Dark regions (d) represent distant cell-substratum contacts. Sections of an outer rim (r) of close contact can be seen along the extending cell margin, at all time points. A region (p) along the rear cell edge (above broken line) becomes more distinct and brighter than its surroundings as retraction proceeds. This may represent the development of focal adhesion precursors, since focal adhesions are commonly found in this location. Bar, $10 \mu \mathrm{m}$.

dynamic features that can change size and shape within seconds during locomotion (Fig. 6). These regions typically undergo cycles of enlargement and bifurcation (Fig. 6A-G) followed by narrowing and shrinkage (Fig. 6H-N). This behavior leads, collectively, to the generation of contact patterns that appear to move forwards with the advancing edge and which may persist for several minutes. Inspection of indi- vidual video frames reveals that the dynamic behaviour of individual close contacts, including the development of patterns, originates at the leading edge (Fig. 6). Changes in size and shape of individual cell-substratum contacts are determined by the relative rates of contact formation at the leading edge and loss of contact at its rear edge (Fig. 7). For example, the outer rim of very close contact can apparently advance with the extending lamella, although is does not actually move with respect to the substratum. This is because formation at the front, and loss at the rear, of this contact region both occur at the same rate as lamellar extension (Fig. 7A). When the rate of contact formation at the front is greater than the rate of loss at the rear, it will elongate perpendicular to the cell margin (Figs 6B-E and 7B). If this process is also occurring parallel to the cell margin then it will become progressively broader, resulting in a ' $\mathrm{v}$ ' shaped region of close contact (Fig. 7C). Typically, enlargement of a region will continue until a critical size is reached. It will then bifurcate, forming two smaller regions which can subsequently undergo enlargement and bifurcation (Figs 6B-G and 7C). When rate of contact formation at its front edge is less than the rate of loss at its rear, the contact will shrink perpendicular to the cell margin (Fig. $6 \mathrm{H}-\mathrm{L})$. If this process is occurring parallel to the leading edge, the contact will also become narrower (Figs 6H-L and 7D). In periodic patterns of contact, the enlargement and bifurcation of one region is frequently accompanied by the shrinkage and narrowing of neighboring regions of contact. This dynamic behaviour results in the maintenance of a periodic pattern of contacts and minimizes local differences in lamellar extension rate.

\section{The relationship between organization of close contacts and cell locomotion}

\section{Cell speed}

In most cells, the pattern of contacts is found within a band whose width perpendicular to the cell edge varies from 5-10 $\mu \mathrm{m}$. The width of this band is greatest beneath regions of the lamella where extension is most rapid and narrowest where extension is slowest (Fig. 2B). To investigate this relationship further, cell speed was measured in cells displaying different patterns of close contacts. Moving keratocytes were observed by IRM for a period of about 2 minutes, while exhibiting the same pattern of contact (Fig. 8A). Keratocytes displaying a simple contact pattern moved at significantly greater speeds $(\sim 8.5 \mu \mathrm{m} /$ minute $)$ than cells with either a periodic pattern $(6.6$ $\mu \mathrm{m} /$ minute $)$ or an irregular one $(3.75 \mu \mathrm{m} /$ minute $)$.

\section{Lamellar extension}

In addition to the spatial organization of close contacts, the persistence of a given contact region over time was found to be an important feature in determining the local rate of lamellar extension. The range of gray scale intensities, together with the local lamellar extension rate was measured within a square region $\left(\sim 5 \mu \mathrm{m}^{2)}\right.$ close to the cell edge, for cells displaying each category of contact pattern (Fig. 8B). The percentage of the total observation period during which the lamella is in very close, intermediate or distant contact with the substratum was obtained using the histogram function of Image 1 software. The most rapid local lamellar extension rate was observed when the subjacent contact is in intermediate contact with the substratum, for the whole period of observation. However, decreased 

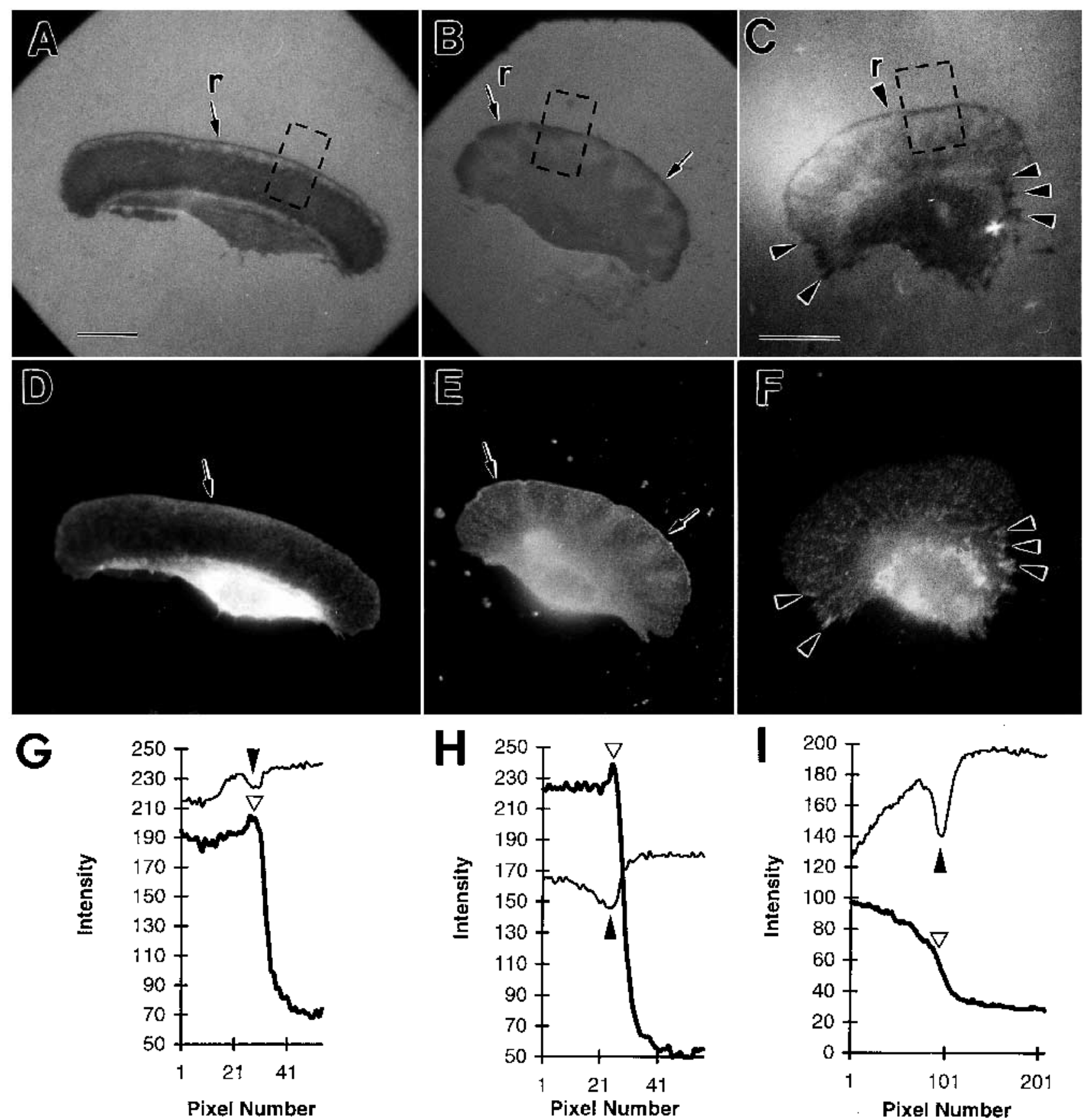

Fig. 4. Paired IRM and immunofluorescence photographs of keratocytes fixed and stained with anti- $\beta 1$ integrin (A and D), anti-talin (B and E) and anti-vinculin antibodies (C and F). $\beta 1$ Integrin and talin immunostaining is found in small foci throughout the lamella of these cells which were fixed using a glutaraldehyde based fixative (protocol A, see Materials and Methods). In both cases, a rim (r) of increased fluorescence corresponds to a narrow region of very close contact along the leading edge as seen by IRM (compare A with D and B with E). This is shown more clearly by the averaged line intensity scans $(\mathrm{G})$ for $\beta 1$ integrin and $(\mathrm{H})$ for talin, taken along the length of the rectangles in A and B, respectively. A pronounced minimum (black arrowhead) in the graphs of light intensity of the IRM images (thin line), marks the position of the rim (r) of very close contact. This coincides exactly with the peak (open triangle) in the graphs of fluorescence intensity (bold line). Note that the increased anti- $\beta 1$ integrin immunofluorescence at the leading cell edge is more diffuse than that seen for anti-talin. Anti-vinculin, immunofluorescence $(\mathrm{F})$ is seen distributed in discrete foci throughout the lamella but is not enriched at the leading edge within the rim of very close contact (C). Averaged line intensity scans (I) taken along the rectangle in $\mathrm{C}$, show a large minimum (black arrowhead) of light intensity (thin line) at the rim (r) of very close contact but without a corresponding peak (open triangle) in the graph for fluorescence intensity (bold line). Vinculin is also located in focal adhesions (arrowheads in C and F) corresponding to small black streaks seen by IRM. This cell was fixed in glutaraldehyde according to protocol C (see Materials and Methods). For clarity, graphs of light intensity for IRM images have been displaced along the $y$-axis, relative to graphs of fluorescence intensity. Bar, $10 \mu \mathrm{m}$. 
Fig. 5. (A,B) Paired IRM (A) and immunofluorescence (B) photographs of a keratocyte stained with anti- $\alpha$ actinin antibodies, following methanol fixation. $\alpha$-Actinin is absent from the three outer rims (r) of very close contact, seen by IRM in A, which are represented by a broken line superimposed on B and also from a band about 5-10 $\mu \mathrm{m}$ wide immediately behind this. Most of the $\alpha$-actinin staining is found within aggregates (open arrows) or densely packed foci (asterisk). Typical $\alpha$-actinin staining is found along stress fibers (arrowheads) within the retracting tail of this keratocyte. Bar in A, $10 \mu \mathrm{m}$. (C,D) Paired IRM (C) and antiFAK (D) immunofluorescence photographs of cells fixed with methanol. FAK is excluded from the outermost rim (r) of very close contact seen in $\mathrm{C}$. Instead FAK is concentrated in numerous, small focal adhesions (small, white arrows) which correspond to fine black streaks by IRM (C). FAK is also found in sparsely distributed foci throughout the lamella. Bar, $10 \mu \mathrm{m}$.

(E,F) Video images of a keratocyte double labeled with rhodamine phalloidin $(\mathrm{E})$ and anti-paxillin anti bodies $(\mathrm{F})$ after fixation in $2.5 \%$ glutaraldehyde according to protocol (c) in Materials and Methods. Paxillin (arrowheads) is concentrated at the tips of actin stress fibers seen in $(\mathrm{E})$ consistent with its primary location in focal adhesions. Bar, $10 \mu \mathrm{m}$.
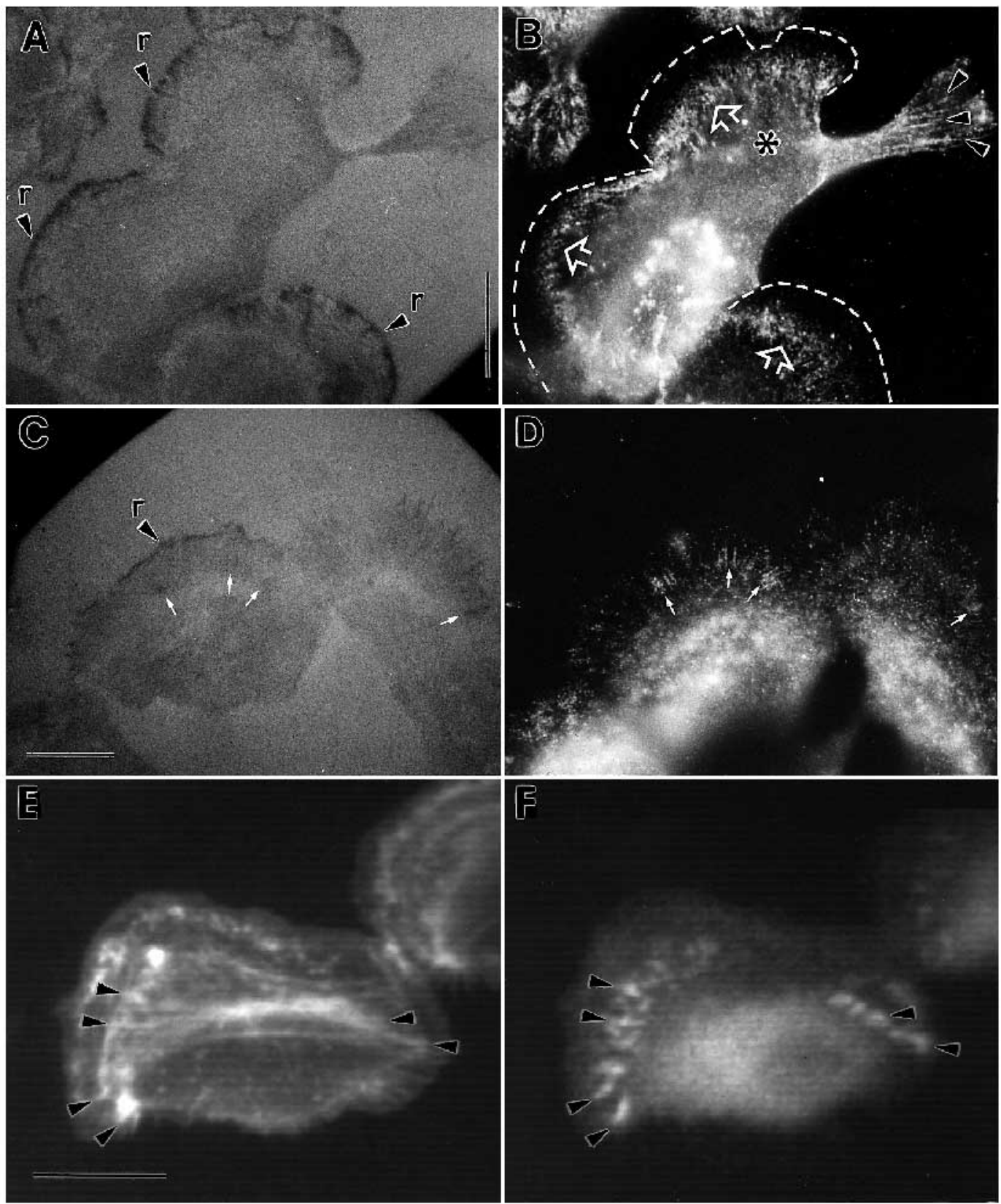

rates of local lamellar extension were observed as its closeness of approach to the substratum became more variable (Fig. 8B). A corresponding decrease in the percentage of the total observation time, spent by a local region of the lamella in intermediate close contact with the substratum was also seen. This is accompanied by an increasingly complex cell shape (compare Fig. 2A and $\mathrm{C}$ ). In this way, the overall rate of lamellar extension is clearly influenced by local differences in rates of close contact formation and loss at the leading cell edge.

\section{Lamellar retraction}

Retraction of the keratocyte lamella also involves a coordinated formation and loss of adhesions at the rear, in the direction of edge movement (Fig. 9). The formation of focal adhesions in keratocytes occurs in response to increasing tension during edge retraction. These usually form in a few seconds, within a region of pre existing close contact, in striking contrast to the slower formation of focal adhesions in fibroblasts. The continuous formation of new focal adhesions together with loss of adhesions within trailing portions of the cell allows retraction to proceed without losing strong attachments to the substratum. This is consistent with the smooth gliding mode of keratocyte locomotion but is in contrast to the common view of lamellar retraction, which is believed to require complete detachment of the ventral cell surface from the substratum.

\section{DISCUSSION}

We have shown that the molecular components of focal adhesions such as $\beta 1$ integrin, talin, vinculin and $\alpha$-actinin are also found in regions of close cell-substratum contact of fish 
Fig. 6. A sequence of images acquired at 4.5 second intervals, taken from a rectangular region that remains fixed with respect to the substratum, during the locomotion of a keratocyte, displaying a periodic pattern of close contacts. The dynamic behaviour of individual close contacts during lamellar extension (bold arrow) is shown.

(A-D) The broadening and bifurcation (b1) of a region of distant contact (white). The front (horizontal arrow) and rear edge (arrowhead) of this contact region, with respect to the substratum in A are superimposed on images B to G. Its apparent forward movement is accompanied by broadening, parallel to the cell edge, resulting in a ' $v$ ' shaped close contact, which then bifurcates (b1, arrowhead). (B-E) The elongation and broadening of a very close contact (dark gray) in B (horizontal arrow) is shown.

Bifurcation (b2,

arrowhead) of this close contact in $\mathrm{E}$ gives rise to an intervening region of distant contact which subsequently elongates and broadens (E-G). The narrowing and shrinkage of a distant contact (vertical arrowhead) is shown in H-L, with respect to a marker (horizontal arrowhead) that remains stationary with respect to the substratum. The merging of two regions of very close contacts in L (paired horizontal arrows) occurs, eliminating an intervening distant contact (L-N). Bar, $10 \mu \mathrm{m}$.

keratocytes. Furthermore, these cells can form focal adhesions containing, in addition to the above molecules, paxillin and FAK. The distribution of these molecules within the keratocyte lamella suggests that close contacts and focal adhesions are related structures formed from a common hierarchy of molecular interactions. We have identified a specialized region of very close contact at the leading edge that is enriched in $\beta 1$ integrin and talin. At this site, the formation of new cell-substratum adhesions occurs simultaneously with lamellar extension. Spatial and temporal differences in the rate of adhesion formation at the cell edge generate distinct patterns of close contact which in turn affect lamellar extension and overall cell speed.

Several lines of evidence suggest that close contacts and focal adhesions are related structures. Firstly, a common feature of adhesion formation is the initial clustering of integrins at the site of cell-substratum contact (Mueller et al., 1989; Mueller and Chen, 1991; Regen and Horwitz, 1992; Lu et al., 1992; Gingell and Owens, 1992). Furthermore, precursors of focal adhesions (Rinnerthaler et al., 1988; Mueller et al., 1989; DePasquale and Izzard, 1991) and 'non focal' adhesions in other cell types (Mueller and Chen, 1991; Arregui et al., 1994) are both primarily composed of integrin and talin molecules. The development of a more stable adhesion is marked by the accumulation of vinculin and its association with the actin cytoskeleton (DePasquale and Izzard, 1987; Rinnerthaler et al., 1988). The continued accumulation of additional molecules such as $\alpha$-actinin, paxillin, and FAK, leads to the formation of a maximally stable or mature focal adhesion. This hierarchy of molecular interactions (Fig. 10) is reflected by the spatial distribution of immunofluorescence staining in keratocytes. Thus newest regions of close contact, containing $\beta 1$ integrin and talin, are found at the leading edge. However, older regions at increasing distances behind the front edge also contain vinculin and $\alpha$-actinin, respectively. It is of interest that highly labile $\alpha$-actinin-containing aggregates, resembling those found in keratocytes, are also found in transformed cells (Stikel and Wang, 1987). This type of adhesion may represent an intermediate between a close contact and a focal adhesion, since $\alpha$-actinin is essential for the formation of stress fibers 


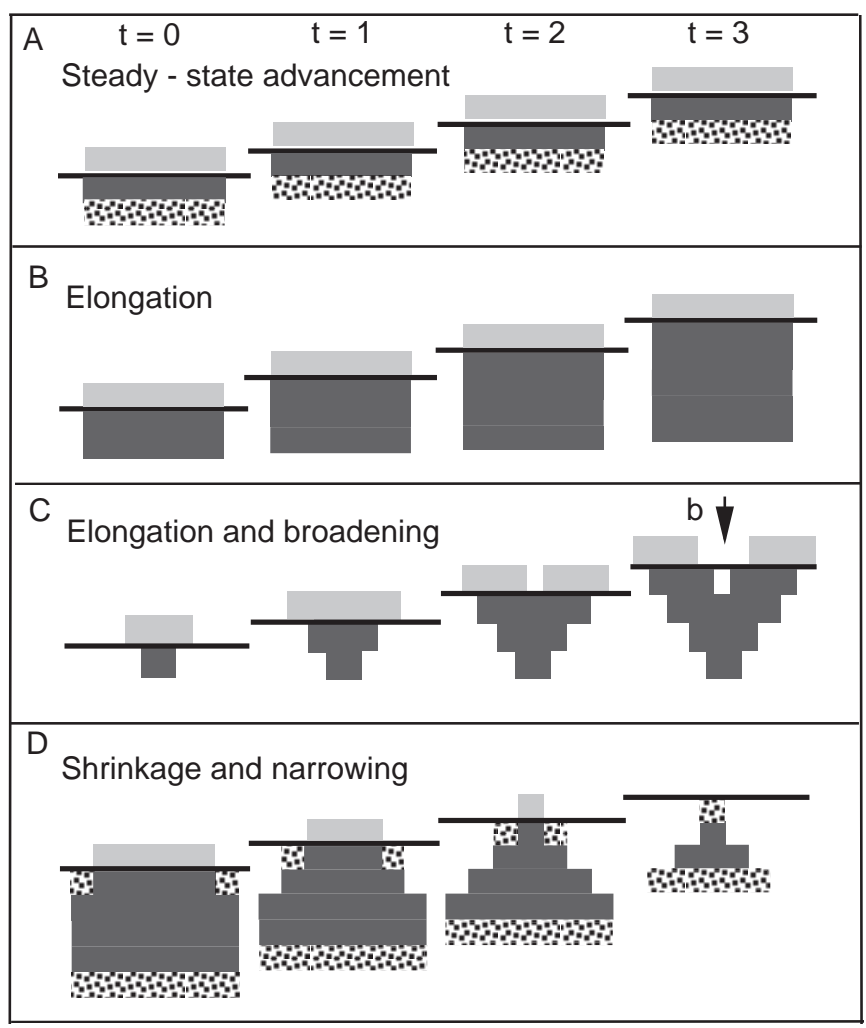

Fig. 7. Diagram illustrating the basic types of dynamic behavior exhibited by regions of close contact (dark gray). The formation of new regions of close contact at the leading edge (bold horizontal line) for each successive time point $(t=1-3)$ is represented by a light gray shaded area. The loss of contact is assumed to occur at its rear edge (stippled squares). The change in shape or apparent movement of a given region of close contact depends on the relative rates of contact formation and loss. (A) Steady-state advancement describes the apparent forward motion of a contact region, in concert with the extending lamella, without any change in shape, e.g. the outer rim of very close contact. This occurs when the rates of contact formation and loss are both equal to the local rate of lamellar extension.

(B) Elongation describes the lengthening of a close contact in the direction of adjacent lamellar extension. This occurs when a new contact is formed perpendicular to and at the same rate as the adjacent lamella extension but with negligible loss of contact at its rear edge. (C) Elongation and broadening of a close contact, leads to the development of a ' $v$ ' shaped structure. This occurs when, in addition to elongation, new contact regions form parallel to the leading edge, without loss of contact from the rear. Bifurcation (b) follows a critical maximum degree of broadening. (D) Shrinkage and narrowing is the converse to that in $\mathrm{C}$. In this case, the rate of new contact formation both perpendicular and parallel to the front edge is less than the rate of contact lost at the rear.

(Meigs and Wang, 1986). Mature focal adhesions, containing a full complement of adhesion components, including paxillin and FAK, are usually found in oldest regions of the lamella that are undergoing retraction.

Another indication that close contacts and focal adhesions are related structures comes from observations that these structures are interconvertible. In fibroblasts, focal adhesions have been shown to form within regions of close contact (Izzard and Lochner, 1980). This is also true for keratocytes at the retracting edges of the cell. A more striking example is that of the
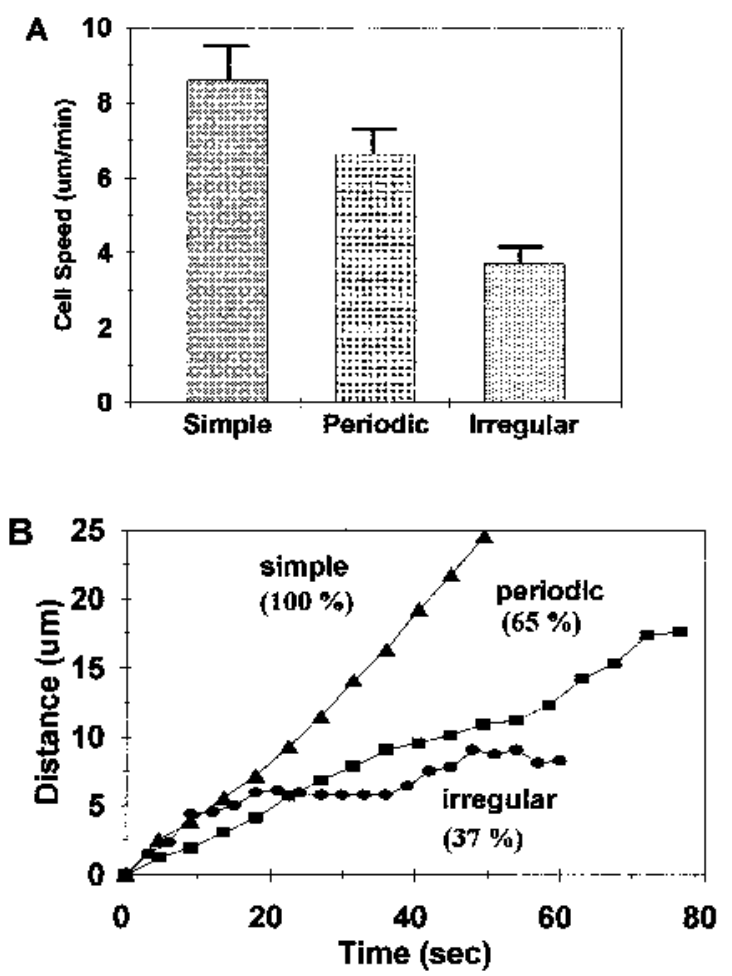

Fig. 8. (A) Histogram of cell speed versus pattern of contacts. The average cell speed was calculated from the mean centroid displacement size taken at 1-2 second intervals for a period of 2-3 minutes. Error bars represent s.e.m. For each type of contact pattern, $n=20$ cells. (B) Graph of the local rate of lamellar extension for each contact pattern, together with the corresponding percentage of the total observation time that the lamella is in intermediate contact with the substratum (in parentheses). Local lamellar extension rate is taken from the centroid displacements of a square region $(\sim 5 \mu \mathrm{m})$ just behind the leading edge whose position remains fixed with respect to the cell outline.

reverse transformation of CHO cells (Leader et al., 1983) where focal adhesions can be induced to form, and then revert back to close contacts.

The diffusive trapping or clustering of adhesion molecule receptors at the cell edge (Gingell and Owens, 1992) is suggested to be a general mechanism for the simultaneous formation of new adhesions and lamellar extension. Recent support for this idea is given by finding that integrin-ligand binding and clustering is required for the formation of new adhesions at the leading edge (Lu et al., 1992) of He La cells. The leading edges of other cell types, besides keratocytes, have also been shown to be enriched in integrins and talin. For example, both these molecules have been found to be concentrated at the leading edge of motile fibroblasts (Izzard, 1988; Memmo and Izzard; 1989; DePasquale and Izzard, 1991). In neuronal cells, integrins are found in point contacts at the cell periphery and at the leading edge of spreading cells (Talwil et al., 1993). Additional evidence for a specialized region of contact at the leading cell edge, comes from IRM observations of an outer rim of close contact in highly motile cell types, such as Rous sarcoma transformed chick fibroblasts (Chen, 1989); macrophages (Heiple et al., 1990); human neutrophils (Yuruker and Niggli, 1992) and the fan shaped amoebae within the genus 


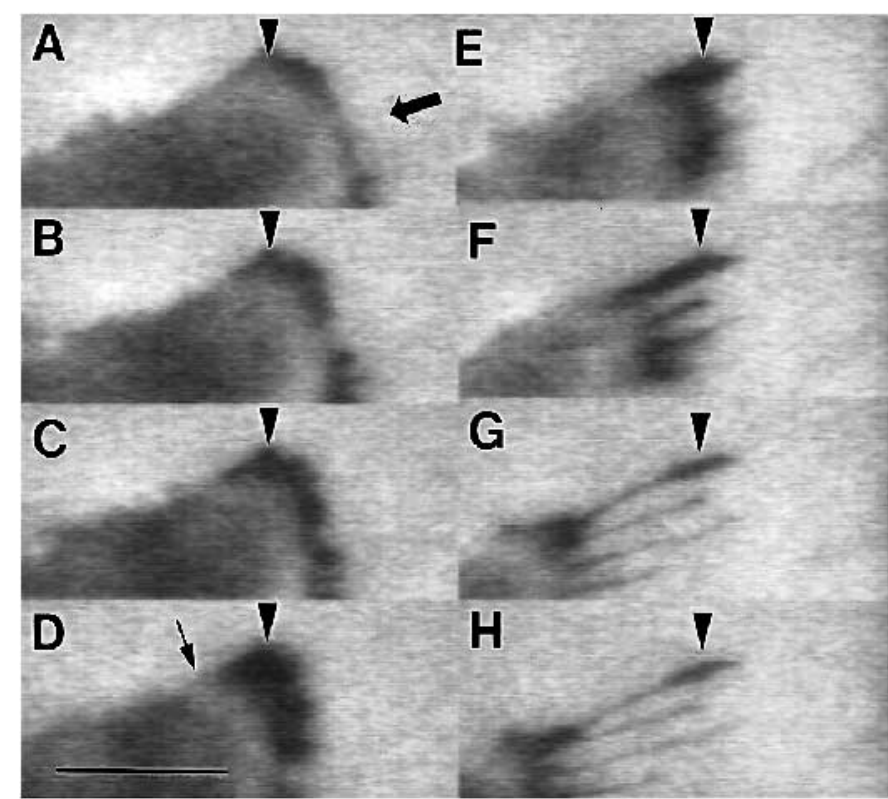

Fig. 9. A sequence of images taken at 4.5 second intervals, from within a rectangle that is stationary with respect to the substratum. This shows the coordinated formation and loss of focal adhesion during the retraction of the rear keratocyte cell margin. (A-D) During retraction, the focal adhesion in A (arrowhead) becomes elongated, due to the formation of new focal adhesion in the direction indicated (arrow). (E-H) As the cell margin retracts, elongation of the focal adhesion continues at the same time as it is lost (arrowhead). Portions of the lamella that remain attached to the substratum (arrowheads) become the tips of retraction fibers F-H. Bar, $10 \mu \mathrm{m}$.

Vanella (J. Lee, unpublished observation). Furthermore, a molecular mechanism exists that can account for specialization of the cell edge for adhesion formation. It has been found that certain membrane glycoproteins, including integrins, are transported forwards on the dorsal cell surface (Kucik et al.,
1991; Sheetz et al., 1990; Schmidt et al., 1995) and become trapped at the leading edge, where they become preferentially attached to the cytoskeleton (Kucik et al., 1991). The finding that actin nucleating proteins, such as ponticulin are localized at the front edge of moving cells (Luna and Hitt, 1992) is an indication that the leading edge also becomes specialized for protrusion. Evidence that the extending edge of keratocytes is the site of net actin polymerisation (Theriot and Mitchison, 1991) together with our present findings, supports the idea that the leading edge is specialized for both the formation of cellsubstratum adhesions and the nucleation of actin filament assembly. According to this idea, anchoring of newly formed actin filaments would occur simultaneously with their assembly. The advantage of this scheme is that it would allow the extending edge to resist any inward directed contractility of the cytoskeleton, leading to a more efficient mode of lamellar extension.

The diffusion trapping of adhesion components at the leading edge and their subsequent release, can explain how patterns of close contacts form in moving keratocytes (Fig. 7). It is also consistent with the finding that close contacts and focal adhesions are interconvertible structures (Fig. 10). According to this idea, trapping of integrins and talin at the leading edge represents the first step in the formation of a cellsubstratum adhesion. As other molecules such as vinculin or $\alpha$-actinin accumulate at the adhesion site, they will become trapped for certain periods of time depending on their respective association and dissociation constants. Conditions that increase the duration of entrapment will increase the stability of the adhesion (Duband et al., 1988) because the accumulation of additional adhesion components such as paxillin and FAK will be favored, leading to the formation of mature focal adhesions. Conversely, it can be seen how by decreasing the duration of entrapment, the stability of the adhesion will be reduced, favoring the development of more labile close cellsubstratum contacts. Thus a key determinant of both the dynamics and type of cell-substratum adhesion is the regulation of adhesion component interactions. At the level of the
Fig. 10. Diagram showing the spatial distribution of simple to more complex (a-e) adhesion types in the keratocyte, as revealed by immunofluorescence. This distribution is consistent with the existence of a hierarchy of molecular interactions in which adhesion formation is initiated at the leading edge (a), close contacts form (b and c), leading to the development mature focal adhesions (d) which later disassemble (e) during lamellar retraction. (a) The entrapment of integrins (red) and actin nucleating factors (gray, triangles) occurs at the leading edge within the outer rim of very close contact. Here, integrin-ligand binding occurs and talin (yellow) accumulates. These molecules associate with the submembraneous actin cortex (light green line) and actin nucleating factors to initiate actin filament assembly. (b) The accumulation of vinculin (blue) is shown within talin and integrin containing close contacts, beneath newly extended portions of the lamella (grey area). (c) The accumulation of $\alpha$-actinin (dark blue) is shown and its association with developing stress fibers (dark green lines) in close contacts within more posterior regions of the lamella. (d) A mature focal adhesion containing FAK (purple) and paxillin (pink) is shown associated with fully developed stress fibers. (e) A focal adhesion disassembly occurs (leaving some $\beta 1$ integrins attached to the substratum) within retracting regions of the lamella.

a

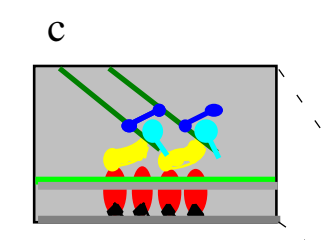

$$
\mathrm{e}
$$

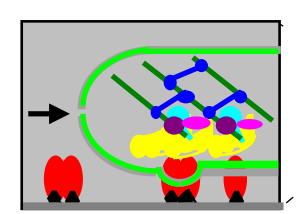

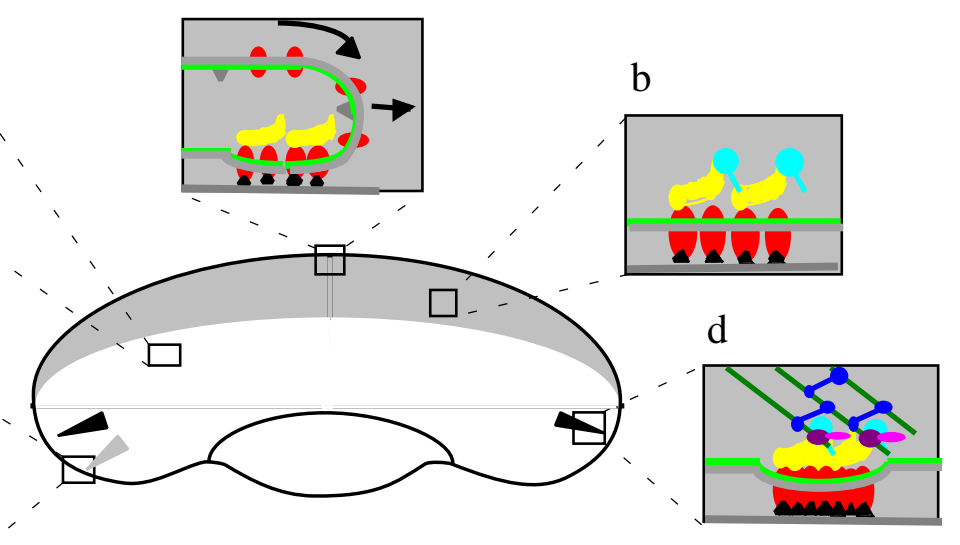


whole cell, such a regulatory mechanism could explain the relationship between cell speed and pattern of cell-substratum contacts. For example, a spatially and temporally uniform pattern of close contacts will permit the most rapid cell locomotion, because extension of all regions of the lamella can occur in a concerted manner. However, as the complexity of the contact pattern increases, so will the variability of local lamellar extension rates. In this way, periodic or irregular contact patterns underlie lamellar extension that is increasingly less coordinated, which in turn will lead to slower cell movement.

Tyrosine phosphorylation of adhesion components plays a dual role in both the formation and disassembly of focal adhesions. The tyrosine phosphorylation of paxillin and FAK has been shown to be required for the formation of focal adhesions (Burridge et al., 1988). Furthermore, focal adhesions can form in response to increased cytoskeletal tension. This has been shown to involve increased tyrosine phosphorylation of adhesion components (Chrzanowska-Wodnicka and Burridge, 1996) which may also affect the strength of molecular linkages between adhesion components and the cytoskeleton. This is consistent with the finding of FAK within focal adhesions at the retracting cell margins of keratocytes, where cytoskeletal tension is high (Lee et al., 1994). Conversely, tyrosine phosphorylation and increased tension are also involved in the disassembly of focal adhesions (Crowley and Horwitz, 1995) during retraction. The phosphorylation of the integrin $\beta 1$ cytoplasmic domain is also believed to be involved, as this inhibits its association with the cytoskeleton (Schmidt et al., 1993). It is likely that close contact formation is governed by the same regulatory mechanisms as focal adhesions, due to their shared structural similarities. Thus the key to rapid cell locomotion lies not only with the molecular composition of cell-substratum adhesions, but also with how the interactions between their components are regulated.

This work is dedicated to the memory of Professor David Gingell. We are grateful to $\mathrm{K}$. Burridge for his gifts of antibodies to focal adhesion components and L. Romer for the polyclonal anti-FAK antibodies. We thank J. Kramer for technical assistance with immunofluorescence studies; M. Reichert and J. Baumeister for assistance with and use of their TIRF microscope and A. Ishihara for critical reading of the manuscript. This work was supported by NIH grant GM35325.

\section{REFERENCES}

Abercrombie, M., Heaysman, J. E. M. and Pegrum, S. M. (1971). The locomotion of fibroblasts in culture. IV. Electron microscopy of the leading lamella. Exp. Cell Res. 67, 359-367.

Arregui, C. O., Carbonetto, S. and McKerracher, L. (1994). Characterization of neural cell adhesion sites: Point contacts are the sites of interaction between integrins and the cytoskeleton in PC12 cells. J. Neurosci. 14, 6967-6977.

Armstrong, P. B. and Lackie, J. M. (1975). Studies on intercellular invasion in vitro using rabbit peritoneal neutrophil granulocytes (PMNS). I. Role of contact inhibition of locomotion. J. Cell Biol. 65, 439-462.

Bereiter-Hahn, J., Fox, C. H. and Thorell, B. (1979). Quantitative reflection contrast microscopy of living cells. J. Cell Biol. 82, 767-779.

Bereiter-Hahn, J., Strohmeier, R., Kunzenbacher, I., Beck, K. and Voth, M. (1981). Locomotion of Xenopus epidermal cells in primary culture. J. Cell Sci. 52, 289-311.

Burridge, K., Fath, K., Kelly, T., Nuckolls, G. and Turner, C. (1988). Focal adhesions: Transmembrane junctions between the extracellular matrix and the cytoskeleton. Annu. Rev. Cell Biol. 4, 487-525.
Chen, W.-T. (1989). Proteolytic activity of specialized surface protrusions formed at rosette contact sites of transformed cells. J. Exp. Zool. 251, 167185.

Chrzanowska-Wodnicka, M. and Burridge, K. (1996). Rho-stimulated contractility drives the formation of stress fibers and focal adhesions. J. Cell Biol. 133, 1403-1415.

Clark, E. A. and Brugge, J. S. (1995). Integrins and signal transduction pathways: the road taken. Science 268, 233-239.

Couchman, J. R. and Rees, D. A. (1979). The behaviour of fibroblasts migrating from chick heart explants: changes in adhesion, locomotion and growth and in the distribution of actomyosin and fibronectin. J. Cell Sci.39, 149-165.

Crowley, E. and Horwitz, A. F. (1995). Tyrosine phosphorylation and cytoskeletal tension regulate the release of fibroblast adhesions. J. Cell Biol. 131, 525-537.

DePasquale, J. A. and Izzard, C. S. (1987). Evidence for an actin-containing cytoplasmic precursor of the focal contact and the timing of incorporation of vinculin at the focal contact. J. Cell Biol. 105, 2803-2809.

DePasquale, J. A. and Izzard, C. (1991). Accumulation of talin in nodes at the edge of the lamellipodium and separate incorporation into adhesion plaques at focal contacts in fibroblasts. J. Cell Biol. 113, 1351-1359.

Duband, J.-L., Nuckolls, G. H., Ishihara, A., Hasegawa, T., Yamada, K. M., Thiery, J. P. and Jacobson, K. (1988). Fibronectin receptor exhibits high lateral mobility in embryonic locomoting cells but is immobile in focal contacts and fibrillar streaks in stationary cells. J. Cell Biol. 107, 1385-1396

Gingell, D. and Owens, N. (1992). How do cells sense and respond to adhesive contacts? Diffusion-trapping of laterally mobile proteins at maturing adhesions may initiate signals leading to local cytoskeletal assembly response and lamella formation. J. Cell Sci. 101, 255-266.

Heath, J. P. and Dunn, G. A. (1978). Cell to substratum contacts of chick fibroblasts and their relation to the microfilament system. A correlated interference-reflexion and high-voltage electron microscope study. J. Cell Sci. 29, 197-212.

Heiple, M. J., Wright, S. D., Allen, N. S. and Silverstein, S. (1990). Macrophages form circular zones of very close apposition to IgG-coated surfaces. Cell Motil. Cytoskel. 15, 260-270.

Hynes, R. O. (1992). Integrins: versatility, modulation and signaling in cell adhesion. Cell 69, 11-25.

Izzard, C. S. and Lochner, L. R. (1976). Cell-to-substrate contacts in living fibroblasts: an interference reflexion study with an evaluation of the technique. J. Cell Sci. 21, 129-159.

Izzard, C. S. and Lochner, L. R. (1980). Formation of cell-to substrate contacts during fibroblast motility: an interference reflexion study. J. Cell Sci. 42, 81-116.

Izzard, C. (1988). Suppport for talin in precursors of focal adhesions. Cell Motil. Cytoskel. 10, 137-142.

Kolega, J., Shure, M. S., Chen, W.-T. and Young, N. D. (1982). Rapid cellular translocation is related to close contacts formed between various cultured cells and their substrata. J. Cell Sci. 54, 23-34.

Kucik, D. F., Kuo, S. C., Elson, E. and Sheetz, M. P. (1991). Preferential attachment of membrane glycoproteins to the cytoskeleton at the leading edge of lamellae. J. Cell Biol. 114, 1029-1036.

Leader, M. W., Stopak, D. and Harris, A. K. (1983). Increased contractile strength and tightened adhesions to the substratum result from reverse transformation of $\mathrm{CHO}$ cells by dibutryl cyclic adenosine monophosphate. $J$. Cell Sci. 64, 1-11.

Lackie, J. M. (1986). Cell Movement and Cell Behaviour. London: Allen Unwin.

Lee, J., Ishihara, A. and Jacobson, K. (1993a). The fish epidermal keratocyte as a model system for the study of cell locomotion. In Cell Behaviour: Adhesion and Motility. SEB Symposium 47 (ed. Jones, G., Wigley, C. and Warn, R.), pp. 73-89. Company of Biologists, Cambridge, UK.

Lee, J., Ishihara, A., Theriot, J. A. and Jacobson, K. (1993b). Principles of locomotion for simple-shaped cells. Nature 362, 167-171.

Lee, J., Leonard, M., Oliver, T. N., Ishihara, A. and Jacobson, K. (1994). Traction forces generated by locomoting keratocytes. J. Cell Biol. 127, 19571964.

Lu, M. L., McCarcon, R. J. and Jacobson, B. (1992). Initiation of HeLa cell adhesion to collagen is dependent upon collagen receptor upregulation, segregation to the basal plasma membrane, clustering and binding to the cytoskeleton. J. Cell Sci. 101, 873-883.

Luna, E. J. and Hitt, A. L. (1992). Cytoskeleton-plasma membrane interactions. Science 258, 955-964. 
Memmo, L. M. and Izzard, C. (1989). $\beta 1$ Subunit of avian integrin is concentrated in nodes along the edge of fibroblast lamellae. J. Cell Biol. 109, 319a.

Meigs, J. B. and Wang, Yu-Li. (1986). Reorganisation of alpha $\alpha$-actinin and vinculin induced by a phorbol ester in living cells. J. Cell Biol. 102, 14301438 .

Mueller, S C., Kelly, T., Dai, M., Dai, H. and Chen, W.-T. (1989). Dynamic cytoskeleton-integrin associations induced by cell binding to immobilized fibronectin. J. Cell Biol. 109, 3455-3464.

Mueller, S. C. and Chen, W.-T. (1991). Cellular invasion into matrix beads: Localisation of $\beta 1$-integrins and fibronectin to the invadopodia. J. Cell Sci. 99, 213-225.

Radice, G. P. (1980). Locomotion and cell-substratum contacts of Xenopus epidermal cells in vitro and in situ. J. Cell Sci. 44, 201-223.

Regen, C. M. and Horwitz, A. F. (1992). Dynamics of $\beta 1$ integrin-mediated adhesive contacts in motile fibroblasts. J. Cell Biol. 119, 1347-1359.

Reichert, W. M. and Truskey, G. A. (1990). Total internal reflection fluorescence (TIRF) microscopy. I. Modelling cell contact region fluorescence. J. Cell Sci. 96, 219-230.

Rinnerthaler, G., Geiger, B. and Small, V. J. (1988). Contact formation during fibroblast locomotion: Involvement of membrane ruffles and microtubules. J. Cell Biol. 106, 747-760.

Schmidt, C. E., Horowitz, A. F., Lauffenburger, D. A. and Sheetz, M. P. (1993). Integrin-cytoskeletal interactions in migrating fibroblasts are dynamic, asymmetric and regulated. J. Cell Biol. 123, 977-991.

Schmidt, C. E., Dai, J., Lauffenburger, D. A., Sheetz, M. P. and Horwitz, A. F. (1995). Integrin-cytoskeletal interactions in neuronal growth cones. $J$. Neurosci. 15, 3400-3407.
Sheetz, M. P., Baumrind, N. L., Wayne, D. B. and Pearlman, A. L. (1990). Concentration of membrane antigens by forward transport and trapping in neuronal growth cones. Cell 61, 231-241.

Schwartz, M. A., Schaller, M. D. and Ginsberg, M. H. (1995). Integrins: emerging paradigms of signal transduction. Annu. Rev. Cell Dev. Biol. 11, 549-600.

Stickel, S. K. and Wang, Y.-L. (1987). Alpha-actinin containing aggregates in transformed cells are highly dynamic structures. J. Cell Biol. 104, 15211526.

Tawil, N., Wilson, P. and Carbonetto, S. (1993). Integrins in point contacts mediate cell spreading: factors that regulate integrin accumulation in point contacts versus focal contacts. J. Cell Biol. 120, 261-271.

Theriot, J. A. and Mitchison, T. J. (1991). Actin microfilament dynamics in locomoting cells. Nature 352, 126-131.

Todd, I., Mellor, J. S. and Gingell, D. (1988). Mapping glass-cell contacts of Dictyostelium amoebae by total internal reflection aqueous fluorescence overcomes a basic ambiguity of interference reflection microscopy. J. Cell Sci. 89, 107-114.

Wehland, J., Osborn, M. and Weber, K. (1979). Cell-to-substratum contacts in living cells: A direct correlation between interference-reflexion and indirect immunofluorescence microscopy using antibodies against actin and $\alpha$-actinin. J. Cell Sci. 37, 257-273

Yuruker, B. and Niggli, V. (1992). Alpha-actinin and vinculin in human neutrophils: Reorganiztion during adhesion and relation to the actin network. J. Cell Sci. 101, 403-414.

(Received 5 September 1996 - Accepted, in revised form, 3 September 1997) 